\title{
Social Distancing as a Health Behavior: County-Level Movement in the United States During the COVID-19 Pandemic Is Associated with Conventional Health Behaviors
}

\author{
Kyle J. Bourassa, $\mathrm{PhD}^{1,2, \oplus} \cdot$ David A. Sbarra, $\mathrm{PhD}^{3} \cdot$ Avshalom Caspi, $\mathrm{PhD}^{1,2,4} \cdot$ Terrie E. Moffitt, $\mathrm{PhD}^{1,2,4}$ \\ Published online: 1 July 2020 \\ (C) Society of Behavioral Medicine 2020. All rights reserved. For permissions, please e-mail: journals.permissions@oup.com.
}

\begin{abstract}
Background Social distancing - when people limit close contact with others outside their household - is a primary intervention available to combat the COVID-19 pandemic. The importance of social distancing is unlikely to change until effective treatments or vaccines become widely available. However, relatively little is known about how best to promote social distancing. Applying knowledge from social and behavioral research on conventional health behaviors (e.g., smoking, physical activity) to support public health efforts and research on social distancing is promising, but empirical evidence supporting this approach is needed.
\end{abstract}

Purpose We examined whether one type of social distancing behavior - reduced movement outside the homewas associated with conventional health behaviors.

Method We examined the association between GPSderived movement behavior in 2,858 counties in USA from March 1 to April 7, 2020 and the prevalence of county-level indicators influenced by residents' conventional health behaviors.

Results Changes in movement were associated with conventional health behaviors, and the magnitude of these associations were similar to the associations among the conventional health behaviors. Counties with healthier behaviors - particularly less obesity and greater physical activity - evidenced greater reduction in movement outside the home during the initial phases of the pandemic in the USA.

1 Center for the Study of Aging and Human Development, Duke University Medical Center, Durham, NC

2 Department of Psychology and Neuroscience, Duke University, Durham, NC

3 Department of Psychology, University of Arizona, Tucson, AZ

4 Institute of Psychiatry, Psychology, \& Neuroscience, King's College London, Strand, London
Conclusions Social distancing, in the form of reduced movement outside the home, is associated with conventional health behaviors. Existing scientific literature on health behavior and health behavior change can be more confidently used to promote social distancing behaviors during the COVID-19 pandemic.

Keywords: COVID-19 $\cdot$ Social distancing $\cdot$ Health behaviors $\cdot$ Smoking $\cdot$ Physical activity $\cdot$ Counties

\section{Introduction}

The SARS-CoV-2 coronavirus originated in Wuhan, China, in late 2019 [1] and spread globally over the first 3 months of 2020. Human-to-human transmission of SARS-CoV-2 was confirmed in January 2020 [2], and the first case in the USA was confirmed on January 24. The outbreak of the disease resulting from SARS-CoV-2 (COVID-19) [3] was declared a pandemic by the World Health Organization on March 11 [4]. By June 11, 2020, the CDC recorded over 2 million cases and 110,000 deaths due to COVID-19 in the USA [5], contributing to over 7.3 million infections and 410,000 deaths worldwide [6].

In March 2020, U.S. public health and government officials began an unprecedented effort to limit the spread of SARS-CoV-2. Owed largely to the lack of effective pharmacological interventions and vaccines, social distancing - a constellation of behaviors that decrease close physical contact among nonhousehold members [7,8]-emerged as the primary mitigation strategy for limiting the spread of the virus. The CDC recommended three specific social distancing behaviors: (i) stay at least $6 \mathrm{ft}$ away from other people, (ii) do not gather in groups, and (iii) stay out of crowded places and avoid mass gatherings [5]. Based on the 
belief that the less people traveled outside their home, the less close physical contact between infected and uninfected individuals would occur, multiple levels of government enacted limitations on business and movement (e.g., closing schools and nonessential businesses, stay-at-home orders [9]) to stymie the spread of the virus. Preliminary research found that government actions restricting movement reduced SARS-CoV-2 infection rates $[8,10]$, though peer-reviewed evidence in the USA is more limited.

Social distancing - also known as physical distancing - is a complex set of human behaviors that will benefit from multidisciplinary study to guide public health implementation [11-13]. The current pandemic is unique in terms of its impact on social and economic activities in the modern USA. Previous viral outbreaks, such as H1N1 [14], did not result in the level of mass behavioral change observed in response to COVID-19. Calls for Americans to limit their movement outside the home is a novel - and in many ways unprecedentedpublic health effort in the modern USA. In light of the limited empirical research focused on such efforts, lessons learned from more comprehensively studied behaviors may be useful in understanding how to best promote social distancing behaviors. Health behaviors like smoking, physical activity, and vaccination rates have been studied by the scientific community for decades, presenting an opportunity to apply relevant theory to promote social distancing $[12,15]$. For example, theories of individual behavior change provide models helping to explain why individuals choose to engage in, or not to engage in, health behaviors related to a number of medical conditions [16]. Ecological models of health behavior also present many levels of influence (e.g., family, community) that can impact people's health-related behaviors [17].

It is tempting to apply social and behavioral research on health behaviors to social distancing based purely on theoretical grounds, but the value of this opportunity is limited by whether social distancing behaviors are affected by the same causal, theoretical variables as conventional health behaviors. Empirical evidence to this end would support the application of health behavior models to social distancing $[15,18]$, particularly given the way health-relevant behaviors tend to correlate $[19,20]$. To the extent that social distancing is associated with more conventional health behaviors, stakeholders could more confidently draw on the broad literature of health behavior change $[12,15,18,21]$ when studying and promoting social distancing.

\section{Present Study}

The present study used objectively measured movement data to examine social distancing behavior in U.S. counties during the COVID-19 pandemic. We hypothesized that counties whose residents evidenced healthier behaviors prior to the pandemic would also show greater reductions in movement during the pandemic. In addition, we predicted associations would remain when controlling for relevant state- and countylevel characteristics.

\section{Methods}

\section{Study Design}

The current study used county-level movement data from March 1 to April 7, 2020 collected by two companiesCuebiq and Streetlight Data. Cuebiq collects movement data from $\sim 15$ million mobile GPS-enabled devices from individuals who consented to have their data collected. These data were then processed and aggregated at the county level to produce daily estimates of movement behavior. Streetlight also uses GPS mobility data and a proprietary algorithm to produce estimates of daily vehicle miles traveled, which are aggregated at the county level. Data from Streetlight were made available starting March 1, 2020, which was defined as the start of the study period. Additional data provided by Citymapper were used for validation purposes. Movement data were combined with county-level health behavior data from the County Health Rankings \& Roadmaps. In the current study, we included all counties in the USA that had movement data from both Cuebiq and Streetlight during the period of interest $(N=2,858)$, which included 48 states and the District of Columbia. Streetlight did not include movement data on counties in Alaska $(n=29)$ and Hawaii $(n=5)$, or 248 counties in the continental USA. Cuebiq did not provide data for two additional counties. Supplemental Data 1 provides the 5-digit county identifier, state, and name for all excluded counties $(n=284)$. We did not have access to any individuallevel data for this study.

\section{Measures}

\section{Movement behavior}

Movement behavior was assessed using data from Cuebiq and Streetlight Data. These companies provided county-level data for the primary outcome variables: daily percentage of people remaining within 1 mile of home and daily vehicle miles traveled. Supplemental Analysis 1 provides an analysis of validity for these measures. For each outcome, we calculated initial change in movement in response to the pandemic by taking the difference between averages over the first 7 days of March and April. Daily percentage of people remaining within 1 mile of home Cuebiq movement data use GPS-enabled devices 
to determine the greatest distance people travel from their homes on any given day $(<330 \mathrm{ft}$, between $330 \mathrm{ft}$ and 1 mile, between 1 and 10 mile, and $>10$ mile). Daily data are aggregated at the county level, resulting in a percentage for each category. We calculated the total daily percentage of people who remained within 1 mile of home. Increases in the percentage of people remaining within 1 mile of home indexed relatively more social distancing behavior.

Daily vehicle miles traveled Streetlight movement data estimate the daily vehicle miles traveled by residents of each U.S. county in the continental USA. Streetlight uses the Cuebiq Mobility Index - continuous movement data calculated separately from the distance people travel from home - and a proprietary algorithm to produce a daily estimate of vehicle miles traveled, which is aggregated by county. We calculated a percentage of daily miles traveled, with the average of the first 7 days of March used as the reference period. Decreases in vehicle miles traveled indexed relatively more social distancing behavior.

\section{Conventional County-Level Health Behaviors}

We used data from the 2019 County Health Rankings \& Roadmaps report to measure county-level indicators influenced by residents' health behavior: smoking, obesity, physical activity, flu vaccination, and mammography screenings. Smoking measured the percentage of the adult population who smoke. Obesity measured the percentage of adults with a body mass index $>30$. We note that strictly speaking, obesity is a physiological risk marker for the development of chronic disease [22] and reflects the cumulative effects of multiple health behaviors, including sedentary and physical activity time as well as diet. We include obesity here as a proxy for these behaviors and other lifestyle factors that give rise to adiposity. Physical activity measured the percentage of adults over 20 reporting no leisure time physical activity. Flu vaccination rates measured the percentage of fee-for-service Medicare enrollees that had an annual flu vaccine. Mammography screening rate measured the percentage of female Medicare enrollees aged 65-74 that received an annual mammography screening. These data were collected from the Behavioral Risk Factor Surveillance System, the CDC Diabetes Interactive Atlas, and Mapping Medical Disparities Tool (for additional information on data sources and methodology, see https://www.countyhealthrankings.org/). All measures were recoded so that higher values reflected a greater percentage of health-protective behaviors. We also averaged health behavior indicators to form a single health behavior composite. The composite evidenced adequate internal reliability $(\alpha=.68)$. Individual health behaviors were all significantly correlated with other health behaviors - effects ranged from small to large, $11 \leq r \mathrm{~s} \leq .70$, all $p s<.001$. The individual health behaviors were all highly correlated with the health behavior composite, $r \mathrm{~s} \geq .61$, $p$ s $<.001$ (Table 1 ).

\section{Demographic and Socioeconomic Characteristics}

The 2019 County Health Rankings \& Roadmaps report included demographic and socioeconomic characteristics at the state and county level. Variables used in the current study included county-level population, median household income, and rurality, as well as percentages of people who were women, non-Hispanic white, older than 65 , and had some college education. State-level population and rurality were also included. These data were collected from the American Community Survey, Small Area Income and Poverty Estimates, and Census Population Estimates.

\section{Data Analysis}

To test our hypotheses, we first examined the correlations between county-level indicators influenced by residents' health behaviors - smoking, obesity, physical activity,

Table 1. Bivariate associations of movement with health behaviors

\begin{tabular}{|c|c|c|c|c|c|c|c|c|}
\hline$N=2,858$ & (1) & $(2)$ & (3) & (4) & $(5)$ & $(6)$ & (7) & $(8)$ \\
\hline Increase in $\%$ of people $<1$ mile of home $(1)$ & 1.0 & & & & & & & \\
\hline Decrease in vehicle miles traveled (2) & .73 & 1.0 & & & & & & \\
\hline Smoking status (3) & .36 & .50 & 1.0 & & & & & \\
\hline Obesity (4) & .41 & .47 & .59 & 1.0 & & & & \\
\hline Physical activity (5) & .47 & .58 & .68 & .70 & 1.0 & & & \\
\hline Flu vaccination rate (6) & .44 & .34 & .20 & .17 & .27 & 1.0 & & \\
\hline Mammography screening rate (7) & .25 & .29 & .27 & .11 & .29 & .46 & 1.0 & \\
\hline Health behavior composite (8) & .55 & .59 & .65 & .61 & .74 & .74 & .70 & 1.0 \\
\hline
\end{tabular}

Note: All health behaviors were coded such that greater values represent more health-protective behaviors. The health behavior composite was an average of the individual health behaviors.

All $p \mathrm{~s}<.001$. 
flu vaccination rate, mammography screening rate, and the overall health behavior composite - and changes in the two movement behavior outcomes. Second, we used mixed effect linear models to test the association between these health behaviors and changes in movement. These models nested counties within states and controlled for a variety of state- and county-level demographic and socioeconomic characteristics. State factors were modeled as level-1 variables and county factors were modeled as level-2 variables in the mixed effect models. Analyses were conducted in MPLUS version 8.3 [23].

\section{Results}

\section{Do Pre-Pandemic Health Behaviors Predict Movement Behavior During the Pandemic?}

U.S. counties with lower rates of smoking and obesity, as well as higher rates of physical activity, flu vaccinations, and mammography screenings, had greater reductions in movement from the first week of March to the first week of April, both in the form of more people remaining within 1 mile of home and people driving fewer vehicle miles per day (Table 1). The bivariate associations among the conventional health behaviors were similar in magnitude to the associations between those health behaviors and changes in movement, which were moderate to large in size (Table 1). The health behavior composite was also associated with changes in both percentage of people remaining within 1 mile of home $(r=.55)$ and vehicle miles people traveled per day $(r=.59)$. Figure 1 illustrates changes in movement in counties categorized using the health behavior composite. When controlling for baseline levels of movement during the first week of March, the health behavior composite explained $30.7 \%$ additional variance in the percentage of people remaining within 1 mile of home and $28.5 \%$ additional variance in the vehicle miles people traveled.

\section{Predicting Change in Movement While Controlling for State- and County-Level Covariates}

We next tested whether health behavior predicted change in movement while accounting for state- and county-level demographic and socioeconomic characteristics in mixed linear models nesting counties within states. Control variables at the state-level included population, rurality, and the presence of a state-wide stay-at-home order. Control variables at the county-level included population, rurality, household income, education, gender, race/ ethnicity, and age. When accounting for these variables, the health behavior composite significantly predicted increases in people remaining within 1 mile of home and decreases in the vehicle miles people traveled (Table 2). The sizes of the standardized effects for the health behavior composite $-\beta=0.11[0.02,0.21]$ for percentage of people remaining within 1 mile of home, $\beta=0.16$ $[0.09,0.24]$ for vehicle miles traveled-were attenuated compared to effect sizes in models that did not include county-level demographic or socioeconomic covariates$\beta=0.58[0.52,0.63]$ and $\beta=0.57[0.51,0.63]$, respectively.

Certain individual health behaviors may be more predictive of changes in movement behavior than others. To test this possibility, we specified mixed linear models for each of the individual health behaviors predicting change in movement behavior independently. Obesity and physical activity were significantly associated with changes in both movement outcomes (Table 3). Flu vaccination rate was significantly associated with decreases in vehicle miles driven, but not with the increase in people remaining within 1 mile of home (Table 3). Smoking and mammography screening rates were not significantly associated with either movement outcome (Table 3). These results suggest that obesity and physical activity were the strongest individual health behavior predictors of reduced movement outside the home.

\section{Demographic and Socioeconomic Covariates Associated With Changes in Movement}

When examining the results of the health behavior composite mixed effect models (Table 2), there were several associations of note at the county level of analysis. First, in terms of socioeconomic status, counties that were wealthier and more educated were more likely to reduce their movement. More urban counties were similarly more likely to reduce their movement outside the home. In terms of demographic factors, counties with a lower percentage of non-Hispanic whites and a higher percentage of adults over the age of 65 were more likely to reduce their movement outside the home, though the sizes of these effects were smaller than those for counties' socioeconomic characteristics. At the state-level of analysis, counties in states that enacted stay-at-home orders had a greater reduction in movement compared with counties without such an order.

\section{Discussion}

The current study examined whether county-level indicators of residents' health behavior were associated with social distancing in response to the COVID-19 pandemic from March 1 to April 7, 2020. Social distancing was assessed using objectively measured movement outside the home collected from GPS-enabled devices and aggregated at the county level $(N=2,858)$ in the USA. 

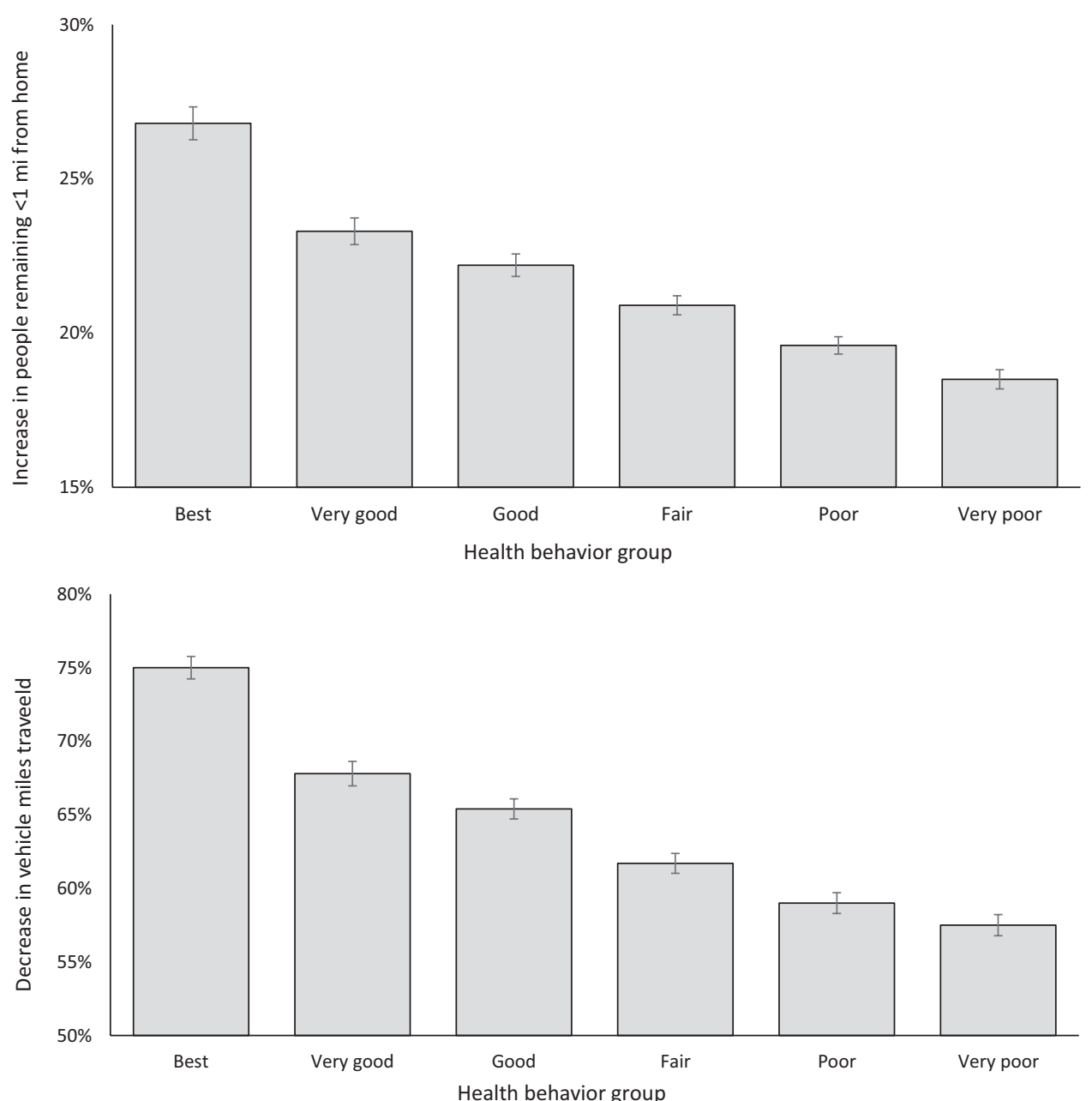

Fig. 1. Change in movement behavior from the first week of March to the first week of April. Counties were categorized by the health behavior composite, which was $z$-scored. Best were $1.0 S D$ or more above the mean $(n=473,16.6 \%)$, Very good $=$ between 0.5 and $1 S D$ $(n=398,13.9 \%)$, Good $=$ between the mean and $0.5 S D(n=532,18.6 \%)$, Fair $=$ the mean and $-0.5 S D(n=544,19.0 \%)$, Poor $=$ between -0.5 and $-1 S D$ below the mean $(n=486,17.0 \%)$, Very poor $<1 S D$ below the mean $(n=425,14.9 \%)$. Error bars represent $95 \%$ confidence intervals.

People in counties with more health-protective behaviors prior to the pandemic reduced their movement to a greater degree during the pandemic than people in counties with fewer health-protective behaviors. The size of these effects were large $-r=.55$ for percentage of people remaining within 1 mile of home, $r=.59$ for vehicle miles traveled, corresponding to roughly $30 \%$ of the variance in these outcomes. In terms of individual conventional health behaviors, rates of obesity and physical activity were particularly robust predictors of movement behavior. The significant associations among individual health behaviors matches well with prior evidence that such behaviors are correlated within individuals $[19,20]$, and extends these results to county-level associations. Although there are calls to apply social and behavioral research [11] to the current crisis on theoretical grounds $[12,15]$, few studies provide empirical evidence to buttress these claims. The findings reported here provide this evidence, which in turn supports application of the wealth of scientific knowledge regarding conventional health behaviors and health behavior change [18, 21, 22] to promote social distancing.

Given the strong associations between conventional health behaviors and changes in movement behavior, a number of theoretical perspectives could have relevance to social distancing. For example, individual models of health behavior could inform our understanding of social distancing [16]. The Health Belief Model highlights how people's perception of threat related to a disease or condition - arising in part from the chance of getting a disease and severity of the disease-helps determine whether people engage in health-protective behavior [16]. Ecological models of behavior change [17, 21] also present a number of principles that could be applied to 
Table 2. Mixed effect model results predicting change in movement behaviors

\begin{tabular}{|c|c|c|c|c|}
\hline \multirow[b]{2}{*}{$N=2,858$} & \multicolumn{2}{|c|}{$\begin{array}{l}\text { Increase in } \% \text { of people remaining }<1 \\
\text { mile from home }\end{array}$} & \multicolumn{2}{|c|}{ Decrease in vehicle miles traveled } \\
\hline & $\beta$ & $95 \% \mathrm{CI}$ & $\beta$ & $95 \% \mathrm{CI}$ \\
\hline \multicolumn{5}{|l|}{ County-level predictors } \\
\hline Health behavior composite & $0.11^{*}$ & {$[0.02,0.21]$} & $0.16^{* *}$ & {$[0.09,0.24]$} \\
\hline March baseline & $-0.32 * *$ & {$[-0.38,-0.26]$} & 0.05 & {$[-0.02,0.12]$} \\
\hline Population size & $0.10^{*}$ & {$[0.02,0.19]$} & -0.01 & {$[-0.07,0.05]$} \\
\hline Rurality & $-0.10^{* *}$ & {$[-0.16,-0.06]$} & $-0.28 * *$ & {$[-0.33,-0.24]$} \\
\hline Household income & $0.46^{* *}$ & {$[0.39,0.54]$} & $0.16^{* *}$ & {$[0.11,0.22]$} \\
\hline Education & $0.23 * *$ & {$[0.18,0.27]$} & $0.37^{* *}$ & {$[0.31,0.43]$} \\
\hline Age & $0.08^{* *}$ & {$[0.03,0.14]$} & $0.07 *$ & {$[0.02,0.13]$} \\
\hline Ethnicity & $-0.17^{* *}$ & {$[-0.27,-0.07]$} & $-0.08^{*}$ & {$[-0.15,-0.01]$} \\
\hline Gender & 0.01 & {$[-0.02,0.03]$} & -0.00 & {$[-0.04,0.04]$} \\
\hline \multicolumn{5}{|l|}{ State-level predictors } \\
\hline Stay-at-home order issued & $0.50 * *$ & {$[0.31,0.69]$} & $0.30^{*}$ & {$[0.06,0.54]$} \\
\hline Population size & -0.01 & {$[-0.18,0.17]$} & -0.00 & {$[0.22,0.21]$} \\
\hline Rurality & 0.11 & {$[-0.21,0.44]$} & 0.18 & {$[-0.21,0.57]$} \\
\hline
\end{tabular}

Models nested counties within states. "March baseline" represents the average of the outcomes over the first week of March. Rurality is coded as percent of the county or state that is rural. Ethnicity is coded as percentage non-Hispanic whites. Education is coded as percent with some college education. Gender is coded as percentage women. "Stay-at-home order issued" assessed whether the state issued a stay-at-home order.

$* p \leq .05$.

$* * p \leq .01$.

Table 3. Associations of movement with individual health behaviors in mixed effect models

\begin{tabular}{|c|c|c|c|c|}
\hline \multirow[b]{2}{*}{$N=2,858$} & \multicolumn{2}{|c|}{$\begin{array}{l}\text { Increase in } \% \text { of people remaining } \\
<1 \text { mile from home }\end{array}$} & \multicolumn{2}{|c|}{ Decrease in vehicle miles traveled } \\
\hline & $\beta$ & $95 \% \mathrm{CI}$ & $\beta$ & $95 \% \mathrm{CI}$ \\
\hline Smoking status & 0.00 & {$[-0.08,0.09]$} & 0.05 & {$[-0.03,0.12]$} \\
\hline Obesity & $0.20 * *$ & {$[0.13,0.26]$} & $0.18 * *$ & {$[0.13,0.23]$} \\
\hline Physical activity & $0.16^{* *}$ & {$[0.08,0.25]$} & $0.21 * *$ & {$[0.15,0.27]$} \\
\hline Flu vaccination rate & 0.05 & {$[-0.01,0.11]$} & $0.05^{*}$ & {$[0.00,0.10]$} \\
\hline Mammography screening rate & -0.03 & {$[-0.09,0.02]$} & 0.02 & {$[-0.05,0.08]$} \\
\hline
\end{tabular}

All health behaviors are coded such that greater values represent more health-protective behaviors.

$* p \leq .05$.

$* * p \leq .01$.

social distaining behaviors. These models emphasize that people's behavior results from a combination of their individual decision-making and context of their family, neighborhood, and community. For example, perceptions of social norms play a powerful role in people's health behaviors, based in part on which behaviors they perceive as normative [24]. The formal and informal groups people identify with (e.g., religious groups, political party identification, neighborhoods) likely impact their perception of social distancing and resulting behavioral choices. Outlining each model of health behavior and how it might apply to social distancing from a theoretical perspective is beyond the scope of this study, but the existing literature presents ample opportunity to make use of accumulated empirical and theoretical knowledge to promote social distancing $[12,15]$ during the current pandemic.

Health behaviors predicted changes in movement when accounting for a variety of demographic and socioeconomic covariates, though the sizes of these 
effects were attenuated. This attenuation is unsurprising given the degree to which demographic and socioeconomic characteristics are associated with health behaviors. For example, socioeconomic disadvantage is associated with smoking, physical inactivity, and obesity [25]. Our results extend these findings to social distancing behavior, as assessed by reduced movement outside the home. Socioeconomic disadvantage at the county-level, assessed by lower county-level household income and education levels, was associated with less of a reduction in movement outside the home. It is likely that the mechanisms proposed to explain socioeconomic disparities in health behaviors - such as differences in community opportunities or resources for health behavior change [25] — are responsible for disparities in countylevel movement. For example, counties with greater socioeconomic disadvantage could have more residents with jobs in essential businesses (e.g., gas stations, grocery stores, healthcare), necessitating more movement outside the home. Alternatively, residents in such counties could have smaller homes and less access to outdoor spaces, which would require more movement to engage in safe recreational activities. The current study did not, however, examine the causes of disparities in movement behavior. The large literature examining socioeconomic disadvantage and health behaviors [25] would be useful in developing methods to reduce disparities during the current pandemic. Effective interventions would be particularly important in socioeconomically disadvantaged counties, given associations of disadvantage with poorer health status and less access to healthcare [26].

We found that county-level measures of health behaviors, demographics, and socioeconomic status were associated with changes in two movement behaviors. However, the extent to which these movement outcomes correspond to the constellation of all possible social distancing behaviors is unknown. For example, we do not know the number of close physical contacts people experienced based on their vehicle miles traveled. Future study of the link between these outcomes and infection or mortality rates from COVID-19 would provide evidence as to which measure of movement behavior is more valuable for informing public health practice during the current pandemic. Regardless of which measure is ultimately more useful, the rapid speed at which these data become available - both Cuebiq and Streetlight Data publish daily estimates with only a 3-day lag periodmakes these measures a promising ongoing method to predict areas that might be at risk of increased rates of infection. These data also have the advantage of avoiding issues with self-presentation bias that might affect selfreported adherence to social distancing behaviors.

The current findings are relevant to public health policy at both a theoretical and practical level. The results provide evidence that interventions attempting to reduce people's movement to limit the transmission of the SARS-CoV-2 coronavirus, and during future pandemics, would benefit from drawing on evidence from past public health interventions targeting conventional health behaviors [18]. Social distancing is likely to remain the primary method of limiting infections to prevent hospitals from becoming overwhelmed until an effective vaccine is developed [27], and maintaining such behaviors will require a wide array of public health actions [29]. Behavioral medicine could play a key role in guiding public health policies for social distancing by promoting methods supported by decades of established theoretical and empirical research. For example, intervening on many levels - such as the individual, neighborhood, and societal level — would likely be more successful than attempts to address only a single level [21, 28]. Even once vaccines are developed, tested, and found to be effective, vaccinating the massive numbers needed to achieve herd immunity - likely in the hundreds of millions in the USA alone - will be a massive undertaking. These efforts could be informed by behavioral medicine approaches used to increase the rate of flu vaccinations [29], particularly among at-risk groups [30-32]. Both prior to a vaccine and once a vaccine is developed, our findings provide empirical evidence supporting the value in applying current evidence on health behavior change (e.g., the NIH-funded Science of Behavior Change) to the promotion of social distancing behaviors.

At a practical level, this work suggests that counties in which people are at greater medical risk of hospitalization and death as a result of COVID-19 are also the counties that reduced their movement outside the home the least in response to the pandemic. The health behaviors associated with less of a reduction in movement, particularly obesity and physical activity, are also associated with increased risk of chronic diseases, including lung diseases [33], diabetes [34], and cardiovascular diseases [35]. These chronic conditions also predict poorer COVID-19 prognosis [35]. Targeting people living in counties with unhealthier behaviors - particularly high levels of obesity and physical inactivity-using more intensive public health interventions could be a cost-effective method to promote social distancing and reduce morbidity and mortality from COVID-19. In addition, hospitals serving catchment areas that include counties with unhealthier behaviors - and likely more people with associated chronic diseases - might expect an outsized proportion of future hospitalizations due to COVID-19 compared with counties with healthier behaviors.

The results of this study should be understood in terms of its limitations. First, the study examined objectively measured movement but did not examine a number of other social distancing behaviors, such as the specific 
number of close-proximity contacts (e.g., $<6 \mathrm{ft}$ ) people had outside their homes. It is possible for people to travel outside their home and maintain social distancing principles. Although we expect other measures of social distancing to be highly correlated with movement outside the home, future work linking movement to viral transmission vectors would benefit our understanding of how movement is tied to risk of infection. Second, the current study was correlational-these methods do not allow us to draw causal conclusions. The association between movement and conventional health behaviors could be explained by a number of alternative variables, such as local infection rates, people's perceptions of their at-risk status, or people's employment status. Importantly, our results do not claim that health behaviors cause differences in movement, but instead tested the extent to which social distancing behaviors were associated with conventional health behaviors, with the goal of providing evidence as to the value in treating social distancing as a conventional health behavior.

Third, this study assessed county-level movement, not individual-level outcomes. The results need to be interpreted cautiously, lest we commit the ecological fallacy $[36,37]$ and assume that what is observed at the county-level can be interpreted in individual-level terms. Future studies would benefit from examining the extent to which conventional health behaviors are associated with social distancing behaviors, as well as how well movement behavior and other social distancing behaviors (e.g., staying $>6 \mathrm{ft}$ away from others, avoiding crowds) correlate within individuals. Ongoing cohort studies that are initiating rapid assessment of social distancing behaviors [38] and possess data on prior health behaviors would be an ideal source for such data. Questions assessing other types of social distancing behaviors in addition to movement outside the home would enable future studies to test the extent to which these social distancing behaviors are associated among individuals.

\section{Conclusions}

The current study examined the association between objectively measured movement outside the home and conventional health behavior indicators during the COVID-19 pandemic. The results of this study provide empirical evidence that social distancing behavior - in the form of reduced movement outside the home-is associated with conventional health behaviors. Public health efforts promoting social distancing would benefit from applying prior theoretical and empirical study of health behavior and health behavior change to social distancing behaviors during the current pandemic.

\section{Supplementary Material}

Supplementary material is available at Annals of Behavioral Medicine online.

\section{Acknowledgments}

The first author received support from National Institute on Aging training grant [grant T32-AG000029]. Aggregated mobility data were provided by three companies: Citymapper (via Citymapper. com), Cuebiq, and Streetlight. Cuebiq is a location intelligence and measurement platform. Through its Data for Good program, Cuebiq provides access to aggregated mobility data for academic research and humanitarian initiatives. These first-party data are collected from anonymized users who have opted-in to provide access to their location data anonymously, through a GDPR-compliant framework. It is then aggregated to the county level to provide insights on changes in human mobility over time. Streetlight made data available for the purpose of nonprofit, public benefit research in response to the COVID-19 pandemic. State- and county-level demographic, socioeconomic, and health behavior data were made available from the County Health Rankings \& Roadmaps (https:// www.countyhealthrankings.org/). The program is a collaboration between the Robert Wood Johnson Foundation and the University of Wisconsin Population Health Institute.

\section{References}

1. Zhou F, Yu T, Du R, et al. Clinical course and risk factors for mortality of adult inpatients with COVID-19 in Wuhan, China: A retrospective cohort study. Lancet. 2020;395:1054-1062.

2. Lai CC, Shih TP, Ko WC, Tang HJ, Hsueh PR. Severe acute respiratory syndrome coronavirus 2 (SARS-CoV-2) and corona virus disease-2019 (COVID-19): The epidemic and the challenges. Int $J$ Antimicrob Agents. 2020;55:105924.

3. World Health Organization. Naming the Coronavirus Disease (COVID-19) and the Virus that Causes It. https://www.who. int/emergencies/diseases/novel-coronavirus-2019/technicalguidance/naming-the-coronavirus-disease-(covid-2019)-andthe-virus-that-causes-it/. Accessibility verified May 14, 2020.

4. World Health Organization. Coronavirus Pandemic. https:// www.who.int/emergencies/ diseases /novel-coronavirus-2019/. Accessibility verified May 14, 2020.

5. Center for Disease Control and Prevention. Coronavirus Disease 2019 (COVID-19). https://www.cdc.gov/coronavirus/2019ncov/. Accessibility verified May 14, 2020.

6. Roser M, Ritchie H, Ortiz-Ospina E, Hasell J. Our World in Data. Coronavirus Pandemic (COVID-19). https:// ourworldindata.org/coronavirus/. Accessibility verified May 14th, 2020.

7. Glass RJ, Glass LM, Beyeler WE, Min HJ. Targeted social distancing designs for pandemic influenza. Emerg Infect Dis. 2006;12:1671-1681.

8. Koo JR, Cook AR, Park M, Sun Y, Sun H, Lim JT, Tam C, Dickens BL. Interventions to mitigate early spread of SARSCoV-2 in Singapore: a modelling study. Lancet Infect Dis. 2020;20:678-688. doi:10.1016/S1473-3099(20)30162-6.

9. Blavatnik School of Government at the University of Oxford. Coronavirus Government Response Tracker. https://www.bsg.ox.ac.uk/research/research-projects/ 
coronavirus-government-response-tracker. Accessibility verified May 14, 2020.

10. Soucy JR, Sturrock SL, Berry I, et al. Estimating Effects of Physical Distancing on the COVID-19 Pandemic Using an Urban Mobility Index. Preprint; doi:10.1101/2020.04.05.2005 4288.

11. Holmes EA, O'Connor RC, Perry VH, et al. Multidisciplinary research priorities for the COVID-19 pandemic: a call for action for mental health science. Lancet Psychiat. 2020;7:547560. doi:10.1016/S2215-0366(20)30168-1

12. West R, Michie S, Rubin GJ, Amlôt R. Applying principles of behaviour change to reduce SARS-CoV-2 transmission. Nat Hum Behav. 2020;4:1-9.

13. Bedford J, Enria D, Giesecke J, et al.; WHO Strategic and Technical Advisory Group for Infectious Hazards. COVID-19: towards controlling of a pandemic. Lancet. 2020;395:1015-1018.

14. Shrestha SS, Swerdlow DL, Borse RH, et al. Estimating the burden of 2009 pandemic influenza A (H1N1) in the United States (April 2009-April 2010). Clin Infect Dis. 2011;52(suppl 1):S75-S82.

15. Bavel JJV, Baicker K, Boggio PS, et al. Using social and behavioural science to support COVID-19 pandemic response. Nat Hum Behav. 2020;4:460-471.

16. Glanz K, Rimer BK, Viswanath K. Health Behavior and Health Education. San Francisco, CA: Josey Bass; 2008.

17. Sallis JF, Owen N, Fisher E. Ecological models of health behavior. In: Glanz K, et al., eds. Health Behavior: Theory, Research, and Practice. San Francisco, CA: Josey-Bass; 2015;5:43-64.

18. Glanz K, Bishop DB. The role of behavioral science theory in development and implementation of public health interventions. Annu Rev Public Health. 2010;31:399-418.

19. Berrigan D, Dodd K, Troiano RP, Krebs-Smith SM, Barbash RB. Patterns of health behavior in US adults. Prevent Med. 2003;36:615-623.

20. de Vries H, van 't Riet J, Spigt M, et al. Clusters of lifestyle behaviors: results from the Dutch SMILE study. Prev Med. 2008;46:203-208.

21. Richard L, Gauvin L, Raine K. Ecological models revisited: their uses and evolution in health promotion over two decades. Annu Rev Public Health. 2011;32:307-326.

22. Nimptsch K, Konigorski S, Pischon T. Diagnosis of obesity and use of obesity biomarkers in science and clinical medicine. Metabolism. 2019;92:61-70.

23. Muthén LK, Muthén BO. Mplus User's Guide. 7th ed. Los Angeles, CA: Muthén \& Muthén; 1998-2012.
24. Reid AE, Cialdini RB, Aiken LS. Social Norms and Health Behavior. Handbook of Behavioral Medicine. Steptoe A, ed. New York: Springer; 2010.

25. Pampel FC, Krueger PM, Denney JT. Socioeconomic Disparities in Health Behaviors. Annu Rev Sociol. 2010;36:349-370.

26. Braveman PA, Cubbin C, Egerter S, Williams DR, Pamuk E. Socioeconomic disparities in health in the United States: what the patterns tell us. Am J Public Health. 2010;100(Suppl 1):S186-S196.

27. Pueyo T. Coronavirus: The Hammer and the Dance. Medium. https://medium.com/@tomaspueyo/coronavirus-the-hammerand-the-dance-be9337092b56. Accessibility verified June 5, 2020.

28. Lee VJ, Chiew CJ, Khong WX. Interrupting transmission of COVID-19: lessons from containment efforts in Singapore. $J$ Travel Med. 2020;27(3):1-5.

29. Betsch C, Böhm R, Chapman GB. Using behavioral insights to increase vaccination policy effectiveness. Policy Insights Behav Brain Sci. 2015;2(1):61-73.

30. Thomas RE, Russell ML, Lorenzetti DL. Systematic review of interventions to increase influenza vaccination rates of those 60 years and older. Vaccine. 2010;28:1684-1701.

31. Lau D, Hu J, Majumdar SR, Storie DA, Rees SE, Johnson JA. Interventions to improve influenza and pneumococcal vaccination rates among community-dwelling adults: A systematic review and meta-analysis. Ann Fam Med. 2012;10:538-546.

32. Corace KM, Srigley JA, Hargadon DP, et al. Using behavior change frameworks to improve healthcare worker influenza vaccination rates: a systematic review. Vaccine. 2016;34:3235-3242.

33. Aubry MC, Wright JL, Myers JL. The pathology of smokingrelated lung diseases. Clin Chest Med. 2000;21:11-35, vii.

34. Wilmot EG, Edwardson CL, Achana FA, et al. Sedentary time in adults and the association with diabetes, cardiovascular disease and death: systematic review and meta-analysis. Diabetologia. 2012;55:2895-2905.

35. Wu Z, McGoogan JM. Characteristics of and important lessons from the coronavirus disease 2019 (COVID-19) outbreak in China. JAMA. 2020;323:1239-1242.

36. Robinson WS. Ecological correlations and the behavior of individuals. Int J Epidemiol. 2009;38:337-341.

37. Curran PJ, Bauer DJ. The disaggregation of within-person and between-person effects in longitudinal models of change. Annu Rev Psychol. 2011;62:583-619.

38. Centre for Longitudinal Studies. UCL's Longitudinal Studies to Survey Thousands About Impact of COVID-19. https:// cls.ucl.ac.uk/ucls-longitudinal-studies-to-survey-thousandsabout-impact-of-covid-19/. Accessibility verified June 5, 2020. 